\title{
MicroRNA-495 targets Notch1 to prohibit cell proliferation and invasion in oral squamous cell carcinoma
}

\author{
LONGKUN LV $^{1 *}$, QIANG WANG ${ }^{1 *}$, YUCHENG YANG ${ }^{1}$ and HONGHAI JI ${ }^{2}$ \\ ${ }^{1}$ Department of Stomatology, Yidu Central Hospital of Weifang, Weifang, Shandong 262550; \\ ${ }^{2}$ Department of Clinical Medicine, Weifang Medical University, Weifang, Shandong 261053, P.R. China
}

Received January 21, 2018; Accepted October 3, 2018

DOI: $10.3892 / \mathrm{mmr} .2018 .9616$

\begin{abstract}
MicroRNAs (miRNAs) are associated with the initiation and progression of oral squamous cell carcinoma (OSCC) by regulating a variety of cancer-associated behaviors . Fully understanding the regulatory mechanism of miRNAs in the pathogenesis of OSCC may provide novel promising approaches for the identification of prognostic biomarkers and therapeutic targets for this particular malignancy. In the present study, reverse transcription-quantitative polymerase chain reaction analysis was performed to detect miRNA (miR)-495 expression in OSCC tissues and cell lines. The effects of miR-495 on the proliferation and invasion of OSCC cells were determined using Cell Counting Kit-8 and Matrigel invasion assays, respectively. The mechanisms underlying the action of miR-495 in OSCC cells were also investigated. Results from the present study revealed that miR-495 expression was downregulated in OSCC tissues and cell lines compare with in adjacent normal tissues and human oral keratinocytes, respectively. Exogenous expression of miR-495 restricted cell proliferation and invasion of OSCC cells in vitro. Notch1 was identified as a direct functional target of miR-495 in OSCC. Furthermore, Notch1 knockdown exhibited inhibitory effects, similar to those induced by miR-495 overexpression in OSCC cells. Restoration of Notch1 expression rescued the suppressive effects of miR-495 on OSCC cell proliferation and invasion. These findings suggested an important role for miR-495 in the regulation of OSCC cell growth and metastasis, at least partly by directly targeting Notch1. In addition, the findings of the present study revealed the potential of miR-495 as a novel therapeutic target for the treatment of patients with OSCC.
\end{abstract}

Correspondence to: Professor Honghai Ji, Department of Clinical Medicine, Weifang Medical University, 7166 Baotong West Road, Weifang, Shandong 261053, P.R. China

E-mail: yi_honghai@yeah.net

*Contributed equally

Key words: oral squamous cell carcinoma, microRNA-495, proliferation, invasion, Notch1

\section{Introduction}

Oral squamous cell carcinoma (OSCC) is one of the most common types of head and neck neoplasms, and accounts for $\sim 3 \%$ of all recently diagnosed tumor patients (1). Despite considerable advances in OSCC treatment, the prognosis of patients with OSCC has demonstrated no notable improvements in recent decades (2). The overall 5-year survival rate of patients with OSCC remains $<50 \%$ (3). The poor prognosis of this disease is mainly due to late stage diagnosis, radiation resistance and recurrence; distant metastases have been reported following combined treatment regimens $(4,5)$. The occurrence and development of OSCC is a complex process involving numerous genetic and epigenetic alterations and dynamic alterations in the expression of coding and non-coding RNAs (6-8); however, the specific mechanism underlying the pathogenesis of OSCC remains unknown. Thus, an enhanced understanding of the mechanisms underlying the carcinogenesis and progression of OSCC is critical to the development of novel and effective therapeutic methods to improve the treatment outcomes of this disease.

MicroRNAs (miRNAs) are a group of naturally occurring, non-coding short RNA molecules of 18 to 25 nucleotides in length (9). miRNAs mediate gene expression at the post-transcriptional or translational levels through their complete or partial complementarity with the 3'-untranslated regions (UTRs) of target genes (10). At present, 2,588 mature human miRNAs have been detected in the human genome and are estimated to modulate the expression of $>30 \%$ of all the protein-coding genes (11). Previously, miRNAs have been reported to be abnormally expressed in almost all types of human cancers (12-14). In addition, studies have indicated that numerous miRNAs are dysregulated in OSCC, including miRNA (miR)-433 (15), miR-195-5p (16), miR-27b (17) and miR-373-3p (18). These aberrantly expressed miRNAs may function as tumor suppressors or as oncogenes, depending on the type of tumor and the biological role of their target genes $(19,20)$. Therefore, the identification of additional dysregulated miRNAs may provide novel insight into the function of miRNAs in the onset and the development of OSCC, and may contribute to the identification of novel therapeutic targets for the treatment of OSCC.

miR-495 was previously demonstrated to be dysregulated in human cancers, including medulloblastoma (21), esophageal 
squamous cell carcinoma (22), glioma $(23,24)$ and osteosarcoma (25); however, the expression, functional roles and underlying molecular mechanism by which miR-495 regulates the progression of OSCC remain unclear. The present study aimed to detect the expression of miR-495 in OSCC tissues and cell lines, and determine its effects on OSCC cells. In addition, the underlying molecular mechanisms by which miR-495 may affect the progression of OSCC were investigated.

\section{Materials and methods}

Tissue samples and cell lines. The present study was approved by the Ethics Committee of the Yidu Central Hospital of Weifang (Weifang, China); written informed consent was obtained from all patients who participated in the present study. Surgically removed OSCC tissues and matched adjacent normal tissues were obtained from 23 patients (15 males, 8 females; age range, 47-69 years) who were diagnosed with OSCC, and who underwent surgical resection at the Yidu Central Hospital of Weifang between February 2015 and December 2016. None of the patients with OSCC had received with chemotherapy, radiotherapy or other specialized treatment prior to surgery. All tissues were immediately snap frozen and stored in liquid nitrogen for further use.

Human oral keratinocytes (HOK) were purchased from ScienCell Research Laboratories, Inc. (San Diego, CA, USA) and maintained in an oral keratinocyte medium (ScienCell Research Laboratories, Inc.). The OSCC cell lines Tca8113, CAL-27 and SCC-9 were acquired from the American Type Culture Collection (Manassas, VA, USA) and cultured in Dulbecco's modified Eagle's medium/F-12 (DMEM/F-12) supplemented with $10 \%$ fetal bovine serum (FBS) and $1 \%$ streptomycin/penicillin mix (all from Gibco; Thermo Fisher Scientific, Inc., Waltham, MA, USA). All cell lines were cultured in a humidified chamber at $37^{\circ} \mathrm{C}$ under $5 \% \mathrm{CO}_{2}$.

Cell transfections. miR-495 mimics and mimic negative controls (miR-NC) were obtained from Guangzhou Rui Bo Biological Technology, Co., Ltd. (Guangzhou, China). The miR-495 mimics sequence was 5'-AAACAAACAUGGUGG ACUUCUU-3' and the miR-NC sequence was 5'-UUCUCC GAACGUGUCACGUTT-3'. To knock down endogenous Notch1 expression, a small interfering RNA (siRNA) targeting Notch1 (si-Notch1) and a negative control (si-NC) were chemically synthesized by Shanghai GenePharma Co., Ltd. (Shanghai, China). The Notch1 siRNA sequence was 5'-ACG AAGAACAGAAGCACAAAGGCGG-3' and the NC siRNA sequence was 5'-UUCUCCGAACGUGUCACGUTT-3'. The Notch1 overexpression plasmid (pcDNA3.1-Notch1) and control empty (pcDNA3.1) plasmid were synthesized at the Chinese Academy of Sciences (Changchun, China). Cells were inoculated into 6-well plates and cultured to $60-70 \%$ confluence. miRNA mimics (100 pmol), siRNAs $(100 \mathrm{pmol})$ or plasmid $(4 \mu \mathrm{g})$ were transfected into cells using Lipofectamine ${ }^{\circledR} 2000$ (Invitrogen; Thermo Fisher Scientific, Inc.), according to the manufacturer's protocols. Co-transfection of miRNA mimics $(20 \mathrm{pmol})$ and plasmid $(0.8 \mu \mathrm{g})$ was also performed using Lipofectamine ${ }^{\circledR} 2000$. Following incubation for $6 \mathrm{~h}$, the transfection mixture was removed and fresh DMEM/F-12 containing 10\% FBS was added into each well. Reverse transcription-quantitative polymerase chain reaction (RT-qPCR) analysis and the Matrigel invasion assay was performed after $48 \mathrm{~h}$. CCK- 8 and western blot analysis were conducted at 24 and $72 \mathrm{~h}$ post-transfection, respectively.

$R T$-qPCR. TRIzol ${ }^{\circledR}$ reagent (Invitrogen; Thermo Fisher Scientific, Inc.) was used to extract the total RNA from tissue samples $(100 \mathrm{mg})$ or cell lines $\left(1.5 \times 10^{6}\right.$ cells), according to the manufacturer's protocols. The quality and concentration of total RNA was determined using a NanoDrop 2000 spectrophotometer (NanoDrop Technologies; Thermo Fisher Scientific, Inc., Pittsburgh, PA, USA). For the detection of miR-495, total RNA (100 ng) was converted into cDNA using the TaqMan microRNA Reverse Transcription kit (Applied Biosystems; Thermo Fisher Scientific, Inc.). miR-495 expression levels were detected by qPCR using the TaqMan microRNA Assay kit (Applied Biosystems; Thermo Fisher Scientific, Inc.). The cycling conditions were as follows: $50^{\circ} \mathrm{C}$ for $2 \mathrm{~min}$ and $95^{\circ} \mathrm{C}$ for $10 \mathrm{~min} ; 40$ cycles of denaturation at $95^{\circ} \mathrm{C}$ for $15 \mathrm{sec}$; and annealing/extension at $60^{\circ} \mathrm{C}$ for $60 \mathrm{sec}$. U6 small nuclear RNA was used as an internal reference for measuring relative miR-495 expression. To analyze Notch1 mRNA expression, cDNA was synthesized from total RNA (100 ng) using a PrimeScript 1st Strand cDNA Synthesis kit (Takara Biotechnology Co., Ltd., Dalian, China), and subjected to amplification with a SYBR ${ }^{\circledR}$ Premix Ex Taq II (Takara Biotechnology Co., Ltd.). All reactions were performed on the Applied Biosystems 7500 Real-Time PCR system (Thermo Fisher Scientific, Inc.). The cycling conditions were as follows: $5 \mathrm{~min}$ at $95^{\circ} \mathrm{C}$, followed by 40 cycles of $95^{\circ} \mathrm{C}$ for $30 \mathrm{sec}$ and $65^{\circ} \mathrm{C}$ for $45 \mathrm{sec}$. $\beta$-actin was used as the internal control for measuring relative Notch1 mRNA expression. The primers were designed as follows: miR-495, 5'-TCCGATTCTTCA CGTGGTAC-3' (forward) and 5'-GTGCAGGGTCCGAGG T-3' (reverse); U6, 5'-GCTTCGGCAGCACATATACTAAAA T-3' (forward) and 5'-CGCTTCACGAATTTGCGTGTCAT-3' (reverse); Notch1, 5'-GTGACTGCTCCCTCAACTTCAAT-3' (forward) and 5'-CTGTCACAGTGGCCGTCACT-3' (reverse); and $\beta$-actin, 5'-AGTGTGACGTGGACATCCGCAAAG-3' (forward) and 5'-ATCCACATCTGCTGGAAGGTGGAC-3' (reverse). Relative gene expression was calculated using the $2^{-\Delta \Delta C q}$ method (26).

Cell Counting Kit-8 (CCK-8) assay. A CCK-8 assay was performed to evaluate the proliferative ability of transfected cells. In brief, transfected cells were collected and seeded $\left(3 \times 10^{3}\right.$ cells/well) in 96 -well plates in triplicate. Following $0,24,48$ and $72 \mathrm{~h}$ of cell culture, $10 \mu \mathrm{l}$ of CCK-8 solution (Beyotime Institute of Biotechnology, Haimen, China) was added directly into each well, and the 96-well plates were incubated at $37^{\circ} \mathrm{C}$ under $5 \% \mathrm{CO}_{2}$ for a further $2 \mathrm{~h}$. The optical density was measured at a wavelength of $450 \mathrm{~nm}$ using a multifunctional microplate reader (Bio-Rad Laboratories, Inc., Hercules, CA, USA).

Matrigel invasion assay. The invasive capacity of transfected cells was assessed using 24-well Transwell chambers (Corning Incorporated, Corning, NY, USA) coated with Matrigel (BD Biosciences, Franklin Lakes, NJ, USA). The 

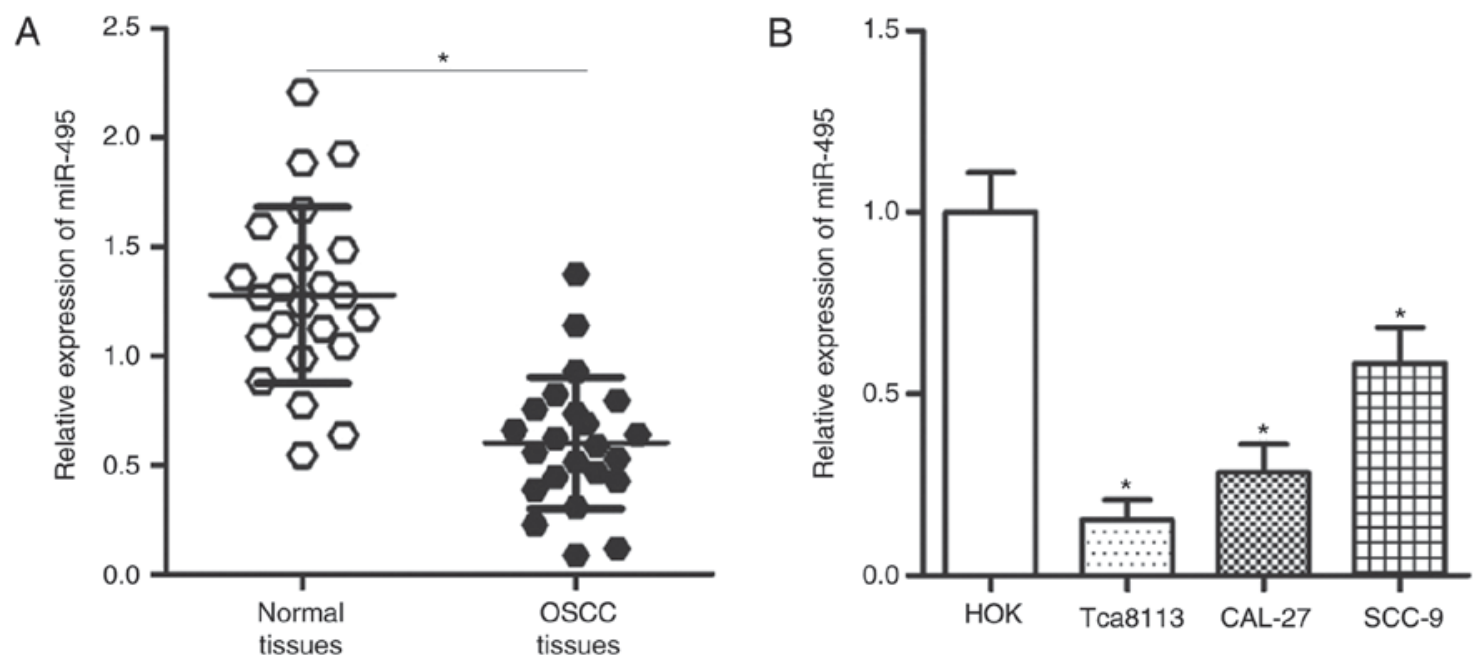

Figure 1. miR-495 is downregulated in OSCC tissues and cell lines. (A) miR-495 expression was detected in 23 pairs of OSCC and matched adjacent normal tissues. *P<0.05 vs. normal tissues. (B) Expression levels of miR-495 in HOK and three OSCC cell lines (Tca8113, CAL-27 and SCC-9). * $<<0.05$ vs. HOK. HOK, human oral keratinocytes; miR, microRNA; OSCC, oral squamous cell carcinoma.

upper compartment of each Transwell chamber was seeded with transfected cells $\left(1 \times 10^{5}\right.$ cells/well) suspended in $200 \mu 1$ FBS-free DMEM/F-12 medium. The lower chambers were filled with $500 \mu \mathrm{l}$ DMEM/F-12 containing 10\% FBS. Cells were incubated in a humidified incubator at $37^{\circ} \mathrm{C}$ under $5 \%$ $\mathrm{CO}_{2}$ for $24 \mathrm{~h}$. The non-invasive cells were removed from the upper chambers with cotton swabs. The invasive cells were then fixed in $95 \%$ ethanol at room temperature for $20 \mathrm{~min}$ and stained in $0.5 \%$ crystal violet at room temperature for $20 \mathrm{~min}$. The invasive cells in the lower chambers were imaged and quantified under an Olympus IX51 inverted microscope (x200 magnification; Olympus Corporation, Tokyo, Japan) in five randomly selected fields per chamber.

miR-495 target prediction and confirmation. Computational analysis was conducted topredictthepotential targetsofmiR-495 using TargetScan (release 7.2; https://www.targetscan.org) and PicTar (http://pictar.mdc-berlin.de). Notch1 was predicted as a potential target of miR-495. The wild-type (WT) and mutant (Mut) putative miR-495-binding sites in the 3'-UTR region of Notch1 were amplified by Shanghai GenePharma Co., Ltd., and inserted into the psiCHECK-2 reporter vector (restriction sites: XhoI and NheI) (Promega Corporation, Madison, WI, USA), which are henceforth referred to as WT-Notch1 and Mut-Notch1, respectively. Cells were cultured in 24-well plates with a density of $1.0 \times 10^{5}$ cells and co-transfected with miR-495 mimics $(50 \mathrm{pmol})$ or miR-NC $(50 \mathrm{pmol})$ and WT-Notch1 $(0.8 \mu \mathrm{g})$ or Mut-Notch1 $(0.8 \mu \mathrm{g})$ using Lipofectamine 2000 . At $48 \mathrm{~h}$ post-transfection, luciferase activity was quantified with a Dual Luciferase Assay kit (Promega Corporation). Firefly luciferase activity was detected with a multifunctional microplate reader (Bio-Rad Laboratories, Inc.), and normalized to Renilla luciferase activity.

Western blot analysis. Cells $\left(1.5 \times 10^{6}\right.$ cells $)$ or tissue samples (250 $\mathrm{mg}$ ) were lysed with radioimmunoprecipitation assay buffer (Beyotime Institute of Biotechnology). Total protein concentration was determined using a BCA Protein Assay kit (Beyotime Institute of Biotechnology). An equal amount of protein $(20 \mu \mathrm{g})$ was separated by $10 \%$ SDS-PAGE and transferred to polyvinylidene fluoride membranes (EMD Millipore, Billerica, MA, USA). Following blocking at room temperature for $2 \mathrm{~h}$ with $5 \%$ fat-free milk in TBS containing $0.1 \%$ Tween-20 (TBST), the membranes were incubated overnight at $4^{\circ} \mathrm{C}$ with primary antibodies against Notch1 (cat. no. ab52627; 1:1,000 dilution; Abcam, Cambridge, UK) or GAPDH (cat. no. ab181603; 1:1,000 dilution; Abcam). Following three washes with TBST, the membranes were incubated with horseradish peroxidase-conjugated goat anti-rabbit secondary antibody (cat. no. ab205718; 1:5,000 dilution; Abcam) at room temperature for $2 \mathrm{~h}$. An Enhanced Chemiluminescence Detection System (Pierce; Thermo Fisher Scientific, Inc.) was used to visualize the signal, according to the manufacturer's protocols. Densitometric analysis of the relative expression levels was performed using Quantity One software version 4.3.0 (Bio-Rad Laboratories, Inc.), and Notch1 expression was normalized to that of GAPDH.

Statistical analysis. Data are presented as the median \pm standard deviation, and were analyzed with SPSS software (version 17; SPSS, Inc., Chicago, IL, USA). Differences between groups were analyzed with a two-tailed Student's t-test or one-way analysis of variance, followed by the Student-Newman-Keuls post hoc test. The correlation between miR-495 and Notch1 mRNA expression levels in OSCC tissues was evaluated with Spearman's correlation analysis. $\mathrm{P}<0.05$ was considered to indicate a statistically significant difference.

\section{Results}

miR-495 expression is downregulated in human OSCC tissues and cell lines. To identify the role of miR-495 in OSCC, the expression levels of miR-495 in 23 pairs of OSCC and matched adjacent normal tissues were analyzed. Results from RT-qPCR revealed that miR-495 expression levels were significantly lower in OSCC tissues compared with the expression levels in the adjacent normal tissues $(\mathrm{P}<0.05$; Fig. $1 \mathrm{~A})$. RT-qPCR was also performed to evaluate the expression levels of miR-495 in 
A

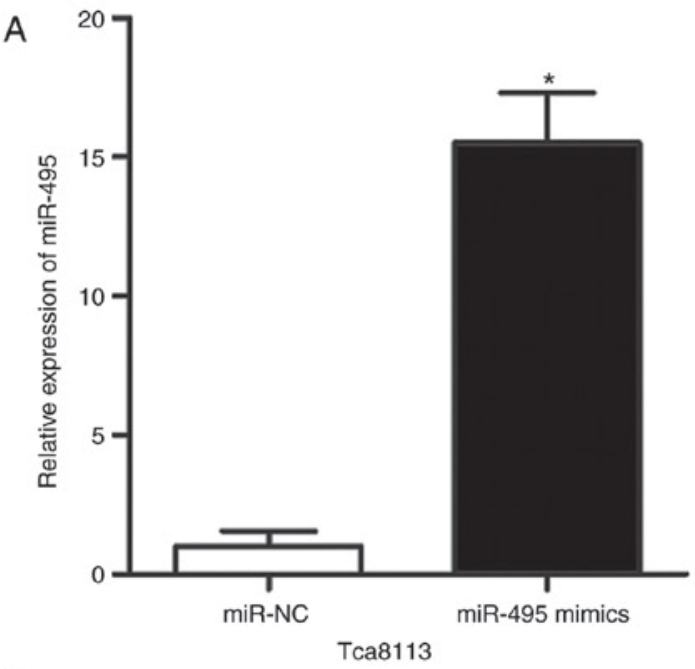

B

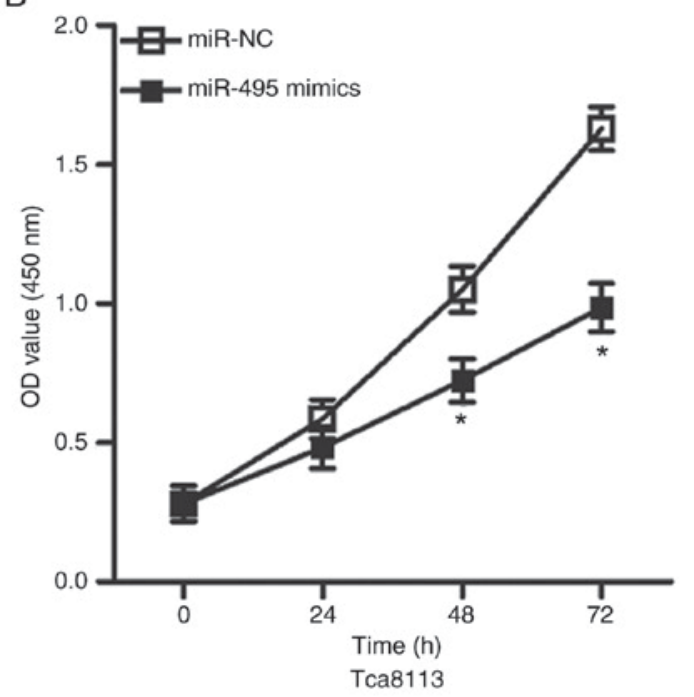

C

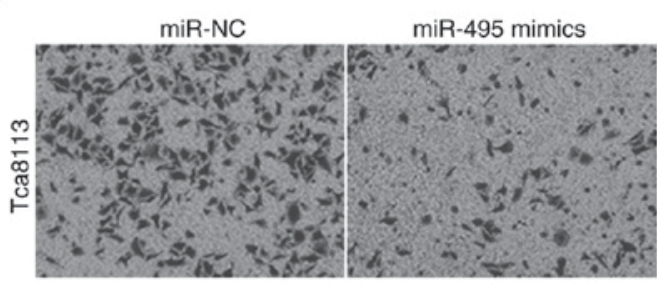

miR-NC

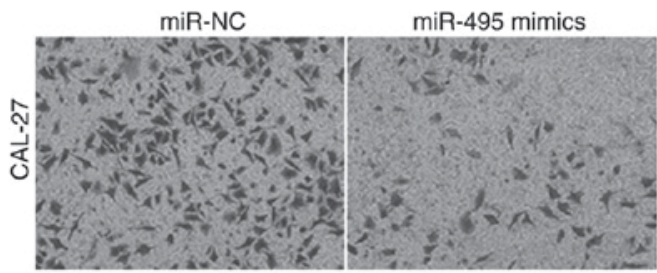

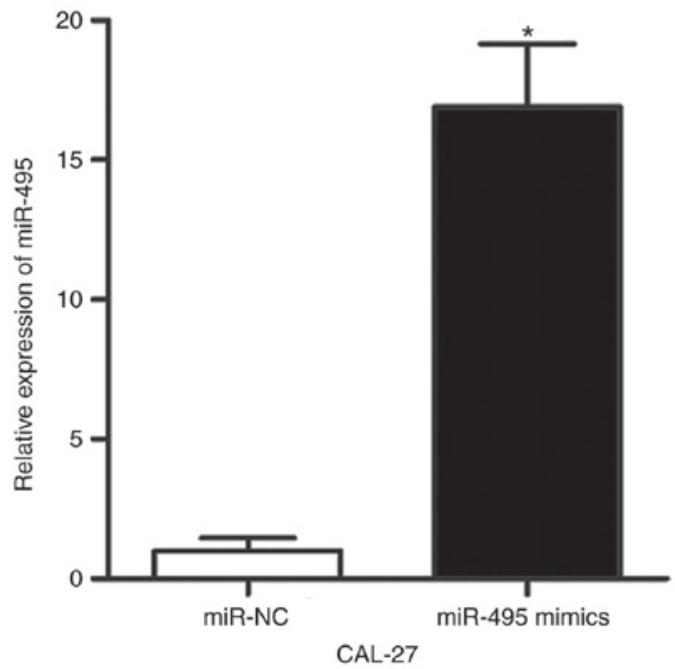
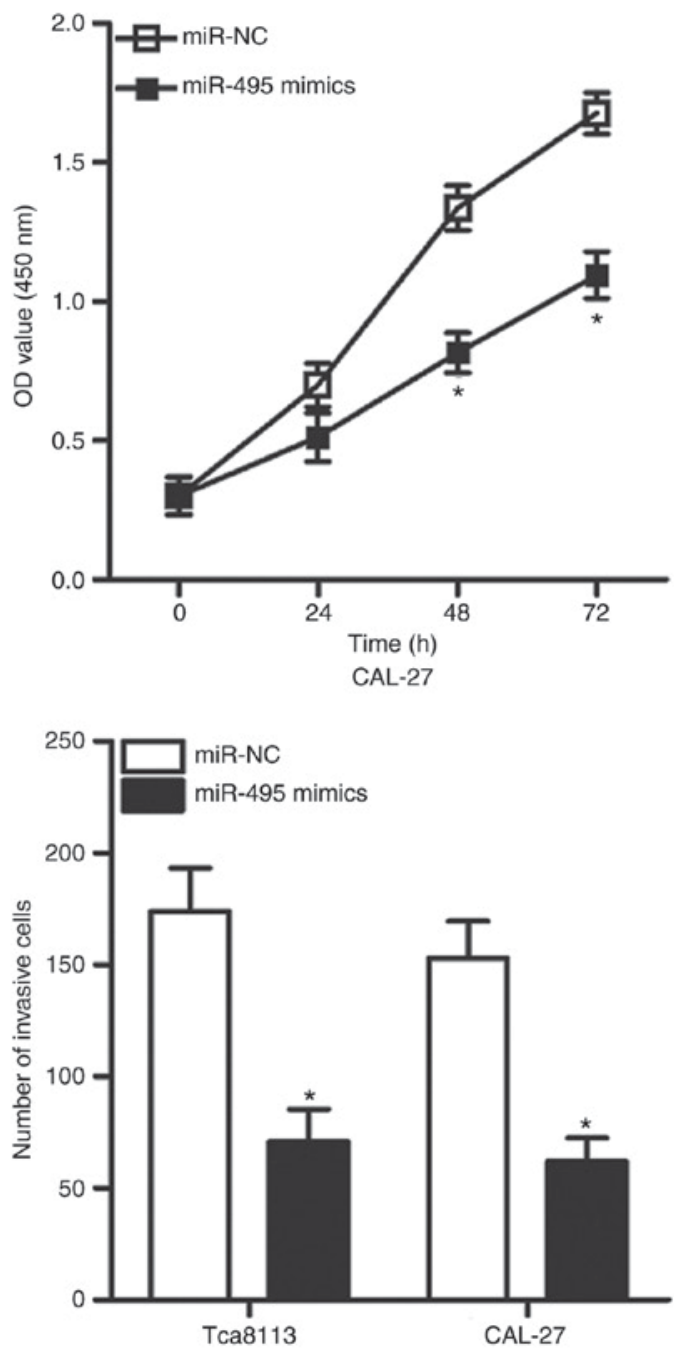

Figure 2. miR-495 overexpression inhibits proliferation and invasion of Tca8113 and CAL-27 cells. (A) Tca8113 and CAL-27 cells were transfected with miR-495 mimics or miR-NC. miR-495 mimics significantly enhanced the expression levels of miR-495 in Tca8113 and CAL-27 cells. (B) Upregulation of miR-495 significantly reduced Tca8113 and CAL-27 cell proliferation at 48 and $72 \mathrm{~h}$ following transfection. (C) Invasive abilities of Tca8113 and CAL-27 cells were significantly suppressed following transfection with miR-495 mimics. * $\mathrm{P}<0.05$ vs. miR-NC. miR, microRNA; NC, negative control; OD, optical density.

three OSCC cell lines, including Tca8113, CAL-27 and SCC-9. Compared with HOK cells, the expression levels of miR-495 were significantly lower in the three OSCC cell lines $(\mathrm{P}<0.05$; Fig. 1B). Tca8113 and CAL-27 cell lines exhibited the lowest relative miR-495 expression among the three OSCC cell lines; therefore, these two cell lines were employed for further analysis. These results suggested that miR-495 may be involved in the development of OSCC. 
A
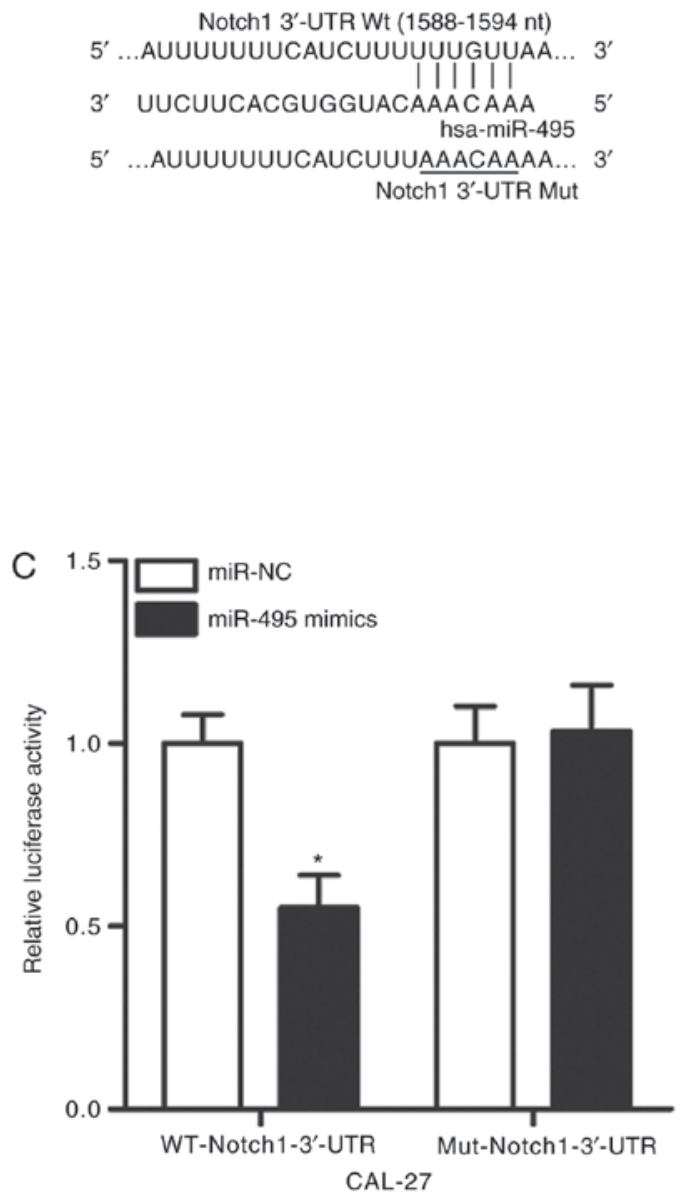

$E$

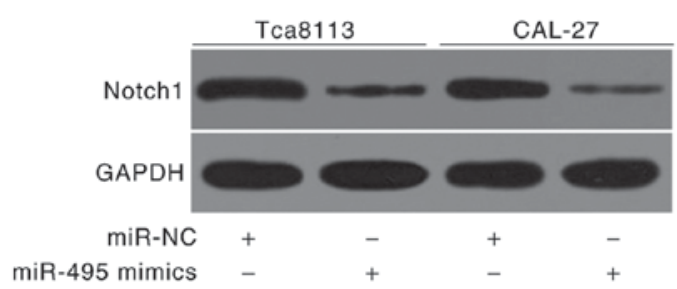

B
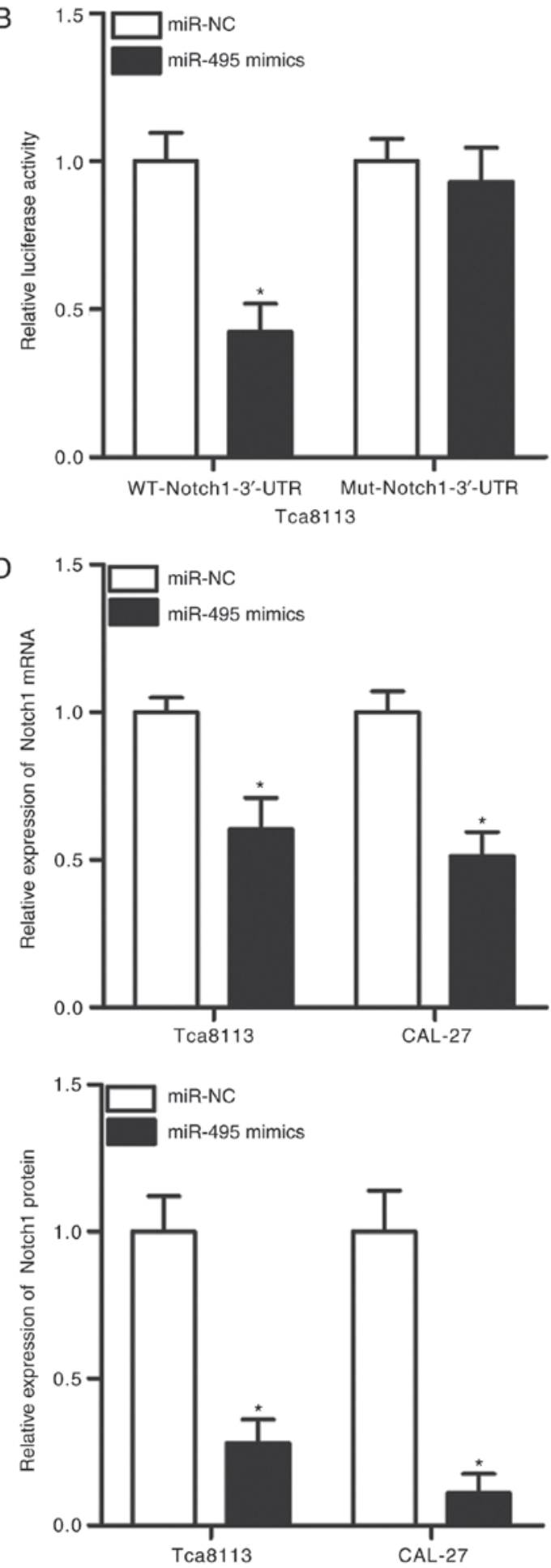

Figure 3. Notch1 is a direct target of miR-495 in oral squamous cell carcinoma. (A) Predicted WT and Mut target sequences for miR-495 on the 3'-UTR of Notch1. (B and C) Dual luciferase reporter assays were used to determine the luciferase activities of the WT-Notch1 or Mut-Notch1 3'-UTR in the presence of miR-495 mimics or miR-NC in (B) Tca8113 and (C) CAL-27 cells. (D and E) Tca8113 and CAL-27 cells were transfected with miR-495 mimics or miR-NC for $48 \mathrm{~h}$, and cells were subsequently collected and subjected to (D) reverse transcription-quantitative polymerase chain reaction and (E) western blot analysis to determine the effects on Notch1 mRNA and protein expression levels, respectively. "P<0.05 vs. miR-NC. miR, microRNA; Mut, mutant; NC, negative control; UTR, untranslated region; WT, wild-type.

Overexpression of miR-495 prohibits the proliferation and invasion of OSCC cells. To investigate whether the dysregulation of miR-495 affected the progression of OSCC, Tca8113 and CAL-27 cells were transfected with miR-495 mimics or miR-NC. RT-qPCR analysis revealed that transfection of miR-495 mimics resulted in a significant increase in miR-495 expression in Tca8113 and CAL-27 cells compared with in the miR-NC group $(\mathrm{P}<0.05$; Fig. $2 \mathrm{~A})$. As demonstrated by the CCK-8 assay, upregulation of miR-495 resulted in significant suppression of Tca8113 and CAL-27 cell proliferation compared with the proliferation rates in the miR-NC group $(\mathrm{P}<0.05$; Fig. 2B). A Matrigel invasion assay was performed to determine 
A

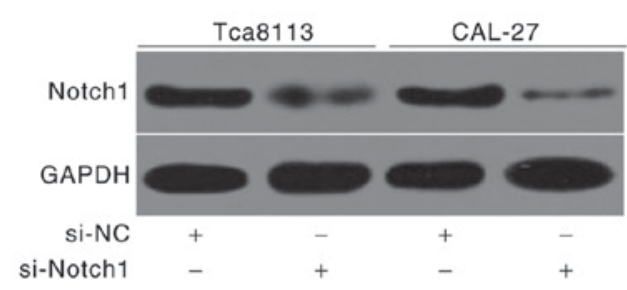

B

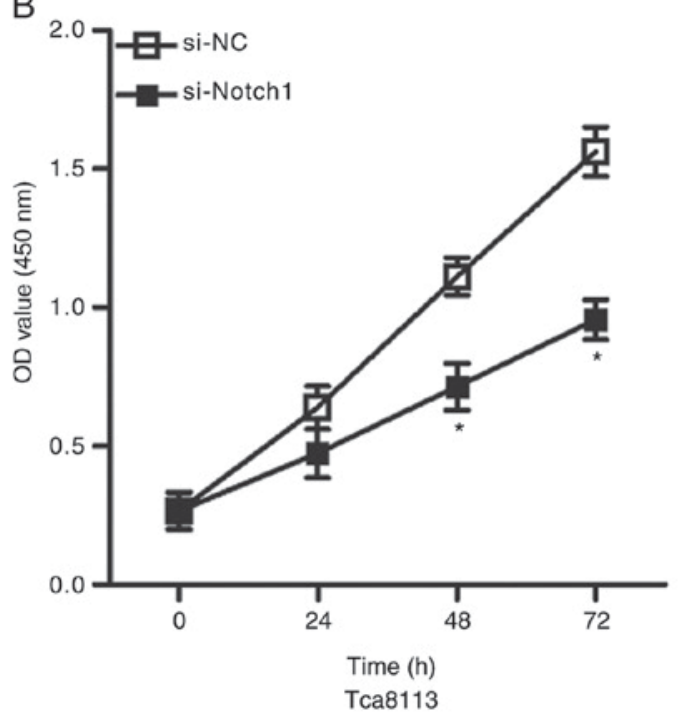

C
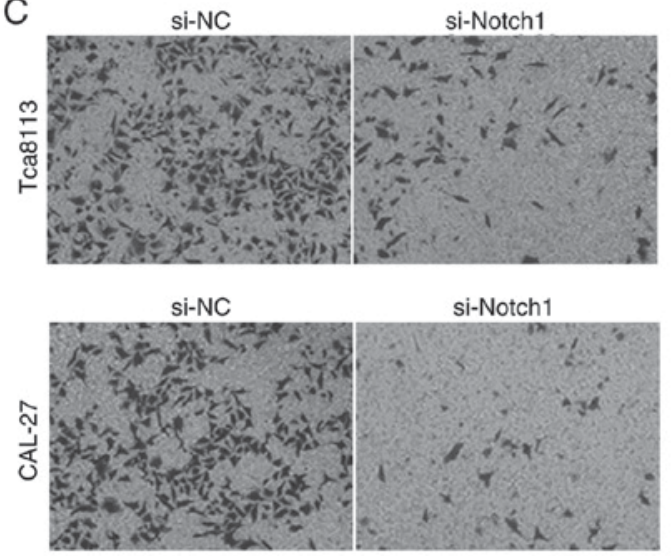
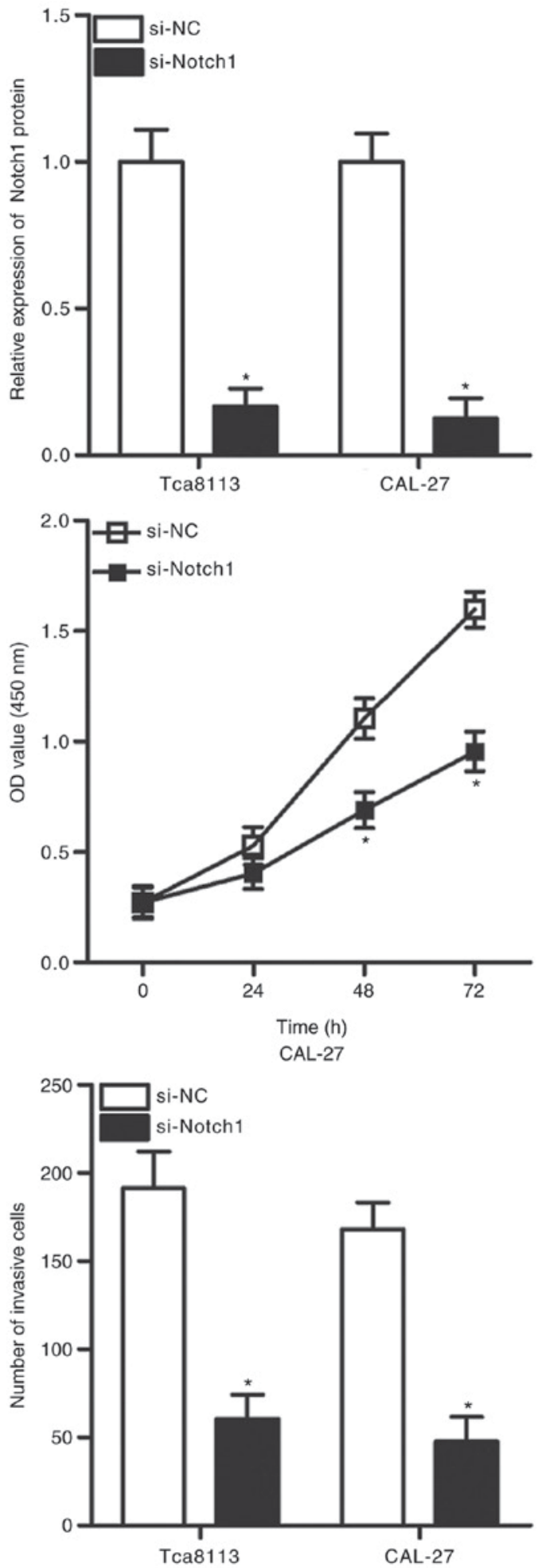

Figure 4. Notch1 knockdown inhibits Tca8113 and CAL-27 cell proliferation and invasion. (A) Transfection with si-Notch1 reduced Notch1 protein expression in Tca8113 and CAL-27 cells. (B) Cell Counting Kit-8 assay results revealed that the proliferative capacities of Tca8113 and CAL-27 cells were significantly decreased at 48 and $72 \mathrm{~h}$ following the knockdown of Notch1 expression. (C) A Matrigel invasion assay demonstrated that the inhibition of Notch1 expression reduced the invasive abilities of Tca8113 and CAL-27 cells. "P<0.05 vs. si-NC. NC, negative control; OD, optical density; si, small interfering RNA.

the effects of miR-495 overexpression on cell invasive ability. The number of invasive cells was significantly decreased in Tca8113 and CAL-27 cells overexpressing miR-495 compared with the miR-NC-transfected group $(\mathrm{P}<0.05$; Fig. $2 \mathrm{C})$. These findings suggested that miR-495 may serve a tumor suppressive role in OSCC.
Notch1 is a direct target of miR-495 in OSCC. To further clarify the mechanism underlying the role of miR-495 in OSCC, computational analysis was conducted to predict the potential targets of miR-495. The 3'-UTR of Notch1 was identified as containing a highly conserved binding site for miR-495 (nucleotides 1588-1594; Fig. 3A). Notch1 was 
previously reported to be involved in the onset and progression of OSCC (27-31) and was therefore selected as a candidate for further confirmation in the present study. A luciferase reporter assay was performed to determine whether the 3'-UTR of Notch1 may be directly targeted by miR-495. The luciferase activity of the reporter containing the WT 3'-UTR of Notch1 was significantly reduced in Tca8113 and CAL-27 cells transfected with miR-495 mimics compared with in the miR-NC group ( $\mathrm{P}<0.05$; Fig. 3B and $\mathrm{C}$ ); however, miR-495 overexpression did not notably affect the luciferase activity of the reporter harboring a mutated miR-495 binding site in the 3'-UTR of Notch1 (Fig. 3B and C). To investigate the role of miR-495 in endogenous Notch1 regulation, RT-qPCR and western blot analyses were performed to detect Notch1 mRNA and protein expression levels, respectively, in Tca8113 and CAL-27 cells following transfection with miR-495 mimics or miR-NC. The results indicated that overexpression of miR-495 significantly decreased the expression levels of Notch1 mRNA and protein in Tca8113 and CAL-27 cells compared with the respective expression levels in the miR-NC-transfected group $(\mathrm{P}<0.05$; Fig. 3D and E). These results indicted Notch1 as a direct target gene of miR-495 in OSCC.

Inhibition of Notch1 mimics the inhibitory effects of miR-495 overexpression in OSCC cells. Providing that Notch1 may be a direct target of miR-495 in OSCC as predicted in the present study, the inhibitory effects of miR-495 in OSCC cell proliferation and invasion may be replicated via the downregulation of Notch1. To confirm this hypothesis, siRNAs targeting Notch1 were transfected into Tca8113 and CAL-27 cells to knock down Notch1 expression. Following transfection, western blot analysis revealed that the expression levels of Notch1 protein were significantly downregulated in Tca8113 and CAL-27 cells transfected with si-Notch1 compared with cells transfected with si-NC ( $\mathrm{P}<0.05$; Fig. 4A). CCK-8 and Matrigel invasion assays indicated that Notch1 knockdown significantly prohibited the proliferation $(\mathrm{P}<0.05$; Fig. 4B) and the invasion rates of Tca8113 and CAL-27 cells compared with si-NC-transfected cells $(\mathrm{P}<0.05$; Fig. 4C). These results corresponded with those obtained following miR-495 overexpression, which further indicated that Notch1 may a functional target of miR-495 in OSCC.

Overexpression of Notchl reverses the suppressive role of miR-495 in OSCC cells. To further investigate whether Notch1 mediates the biological role of miR-495 in OSCC cells a series of rescue experiments were performed. miR-495-overexpressing Tca8113 and CAL-27 cells were transfected with pcDNA3.1-Notch1 or the pcDNA3.1 empty plasmid control. Western blot analysis confirmed that the significantly decreased expression levels of Notch1 protein induced by miR-495 overexpression were recovered by co-transfection with pcDNA3.1-Notch1 in Tca8113 and CAL-27 cells $(\mathrm{P}<0.05$; Fig. 5A). Functional experiments indicated that the restoration of Notch1 expression rescued the suppressive effects of miR-495 mimics on proliferation $(\mathrm{P}<0.05$; Fig. 5B) and invasion $(\mathrm{P}<0.05$; Fig. 5C) of Tca8113 and CAL-27 cells. These results suggested that miR-495 may serve a tumor-suppressing role in OSCC cell proliferation and invasion, at least partly through the inhibition of Notch1 expression.

\section{Discussion}

miRNAs have been associated with the initiation and progression of OSCC by regulating a variety of cancer-associated behaviors, including cell proliferation, apoptosis, cell cycle, invasion, metastasis and angiogenesis (32-34). Therefore, fully understanding the regulatory mechanism of miRNAs in the pathogenesis of OSCC may provide novel, promising approaches for the development of prognostic biomarkers and therapeutic targets. In the present study, it was demonstrated that miR-495 was downregulated in OSCC tissues and cell lines. Exogenous expression of miR-495 inhibited the proliferative and invasive abilities of OSCC cells. In addition, Notch1 was determined to be a direct target gene of miR-495 in OSCC cells. Results from the present study also demonstrated that Notch1 knockdown imitated the inhibitory effects of miR-495 on OSCC cells. Furthermore, restoration of expression of Notch1 counteracted the suppressive effects of miR-495 overexpression on OSCC cell proliferation and invasion. The results of the present study suggested that miR-495 may act as a tumor suppressor in OSCC, and may therefore be considered as a therapeutic target for patients with OSCC.

Downregulation of miR-495 is frequently observed in various types of human cancer (21-24). For example, miR-495 is downregulated in medulloblastoma tissues and cell lines; patients with medulloblastoma and decreased expression levels of miR-495 exhibit poorer prognosis compared with those expressing higher miR-495 levels (21). Furthermore, miR-495 has been reported as an independent predictor of overall survival in patients with medulloblastoma (21). In esophageal squamous cell carcinoma, the expression levels of miR-495 were reported to be reduced in tumor tissues and significantly associated with lymph node metastasis, invasion and tumor, node and metastasis (TNM) stage (22). miR-495 was observed to be expressed at low levels in glioma $(23,24)$, osteosarcoma (25), colorectal $(35,36)$, gastric (37-39), prostate (40) and endometrial cancers (41), and renal cell carcinoma (42). miR-495 has been reported to be highly expressed in bladder (43) and breast (44) cancers; miR-495 upregulation in bladder cancer has been strongly correlated with larger tumor sizes, advanced TNM stages and lymph node metastasis (43). These observations suggested that the expression pattern of miR-495 in human cancers is tissue specific, and may therefore be employed as a diagnostic biomarker and prognostic predictor for patients with these types of cancer.

miR-495 has been associated with the malignant progression of several types of human malignancy. For instance, upregulation of miR-495 has been reported to lead to the reduction of cell proliferation and invasion in glioma $(23,24)$. Another study revealed that restoration of miR-495 expression attenuated cell proliferation and invasion, and promoted the apoptosis of osteosarcoma cells (25). Ectopic expression of miR-495 was demonstrated to prohibit proliferation, colony formation, metastasis and epithelial-mesenchymal transition (EMT), and induce the apoptosis of colorectal cancer cells $(35,36)$. Numerous studies have also demonstrated that miR-495 acts as a tumor suppressor in gastric cancer by regulating cell growth, motility and chemotherapy resistance $(37-39,45)$. Overexpression of miR-495 suppressed prostate cancer cell proliferation, migration and invasion 
A

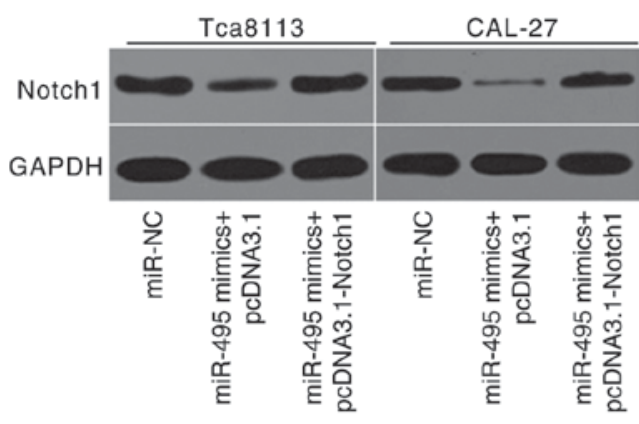

B

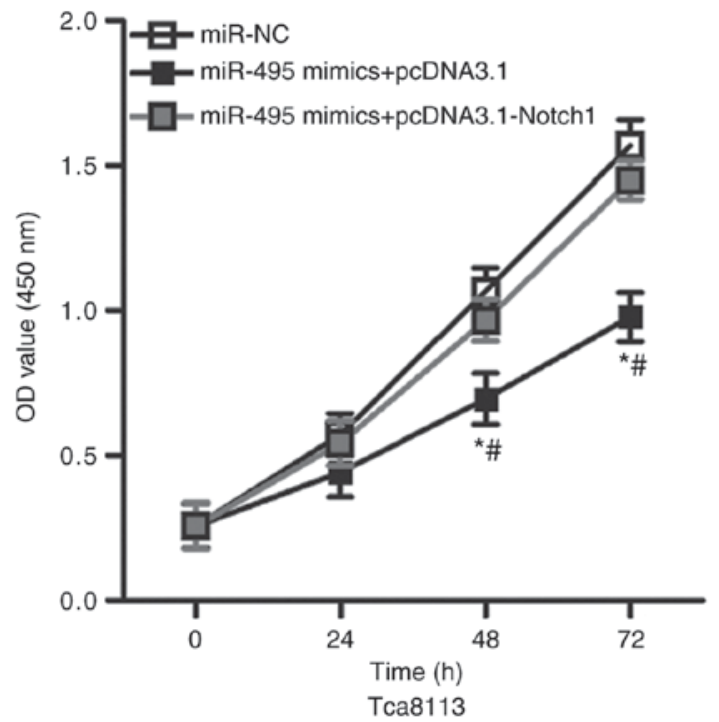

C

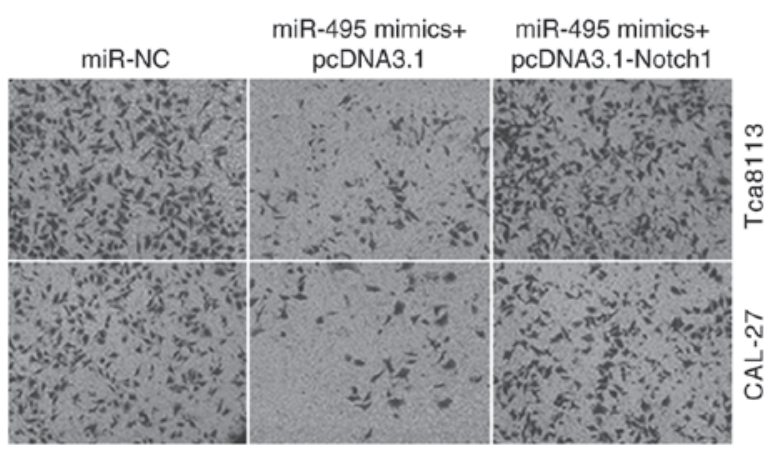

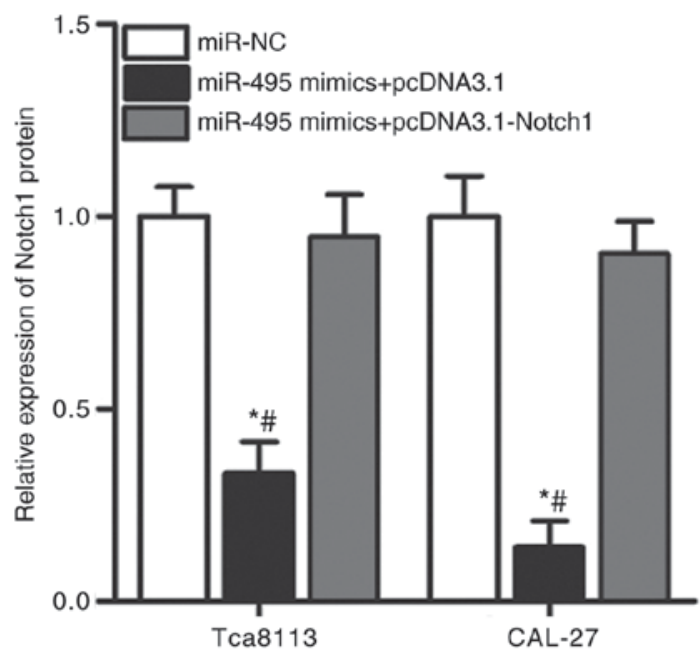
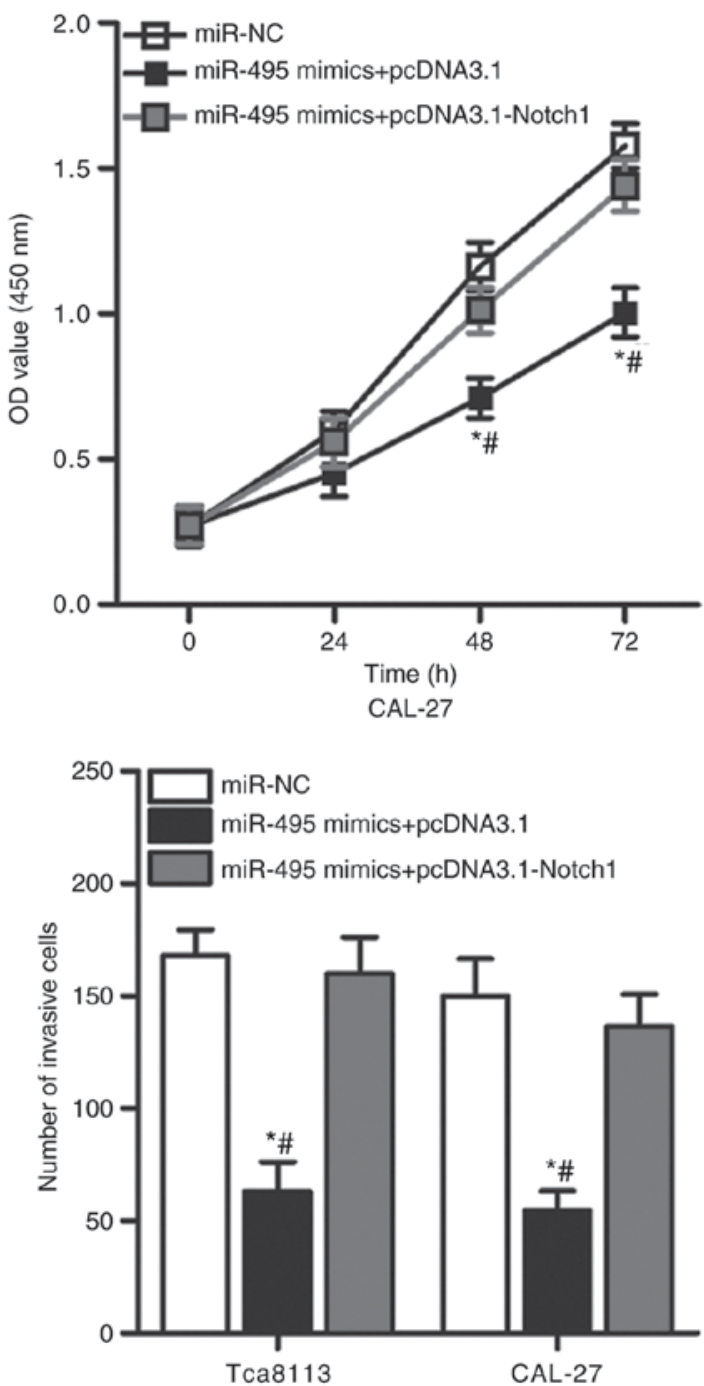

Figure 5. Ectopic expression of Notch1 eliminates the inhibitory effects of miR-495 overexpression on Tca8113 and CAL-27 cell proliferation and invasion. (A) miR-495-overexpressing Tca8113 and CAL-27 cells that were co-transfected with pcDNA3.1-Notch1 overexpression vector or pcDNA3.1 empty vector were subjected to western blot analysis for the detection of Notch1 protein expression. (B) Overexpression of Notch1 reversed the effects of miR-495 overexpression on Tca8113 and CAL-27 cell proliferation. (C) A Matrigel invasion assay revealed that invasive abilities were recovered in miR-495 mimics-transfected Tca8113 and CAL-27 cells following the co-transfection with pcDNA3.1-Notch1. ${ }^{*} \mathrm{P}<0.05$ vs. miR-NC; ${ }^{\#} \mathrm{P}<0.05$ vs. miR-495 mimics + pcDNA3.1. miR, microRNA.

in vitro, and tumor growth in vivo (40). Restoration of the expression of miR-495 reduces endometrial cancer cell growth and migration, and increases apoptosis (41) and decreased renal cell carcinoma cell proliferation and migration, and induced G0/G1 phase arrest (42). Additionally, miR-495 has been reported to serve as an oncogene in bladder (43) and 
breast cancers $(44,46)$, by promoting cell proliferation, colony formation, migration and invasion in vitro, and tumorigenesis in vivo. These findings suggested that miR-495 may possess the potential as an effective target for cancer therapy.

Numerous miR-495 targets have been previously identified, including: Cyclin-dependent kinase 6 and MYB in glioma $(23,24)$; high mobility group (HMG) nucleosome binding domain 5 in osteosarcoma (25); family with sequence similarity 83 member D and Annexin A3 in colorectal cancer $(35,36)$; phosphatase of regenerating liver-3, HMG AT-hook 2 and ATP binding cassette subfamily B member 1 in gastric cancer $(38,39,45)$; protein kinase $\mathrm{B}$ in prostate cancer (40); forkhead box C1 in endometrial cancer (41); stabilin 1 in renal cell carcinoma (42); phosphatase and tensin homolog in bladder cancer (43); and junctional adhesion molecule-A in breast cancer (44). Notch1, a member of the Notch receptors, was predicted as a novel direct target gene of miR-495 in OSCC in the present study. Notch1 was reported to be overexpressed in OSCC, which was correlated with the T-stage, clinical stage, differentiation, lymph node metastasis, depth of invasion and locoregional recurrence (27-29). Notch1 activation has been reported to promote OSCC cell proliferation, apoptosis, migration, invasion and EMT (29-31). These data suggested that targeting Notch1 may be a useful approach for treating OSCC.

In conclusion, to the best of our knowledge, the present study demonstrated for the first time that miR-495 was downregulated in OSCC, which may exert an inhibitory effect on the proliferation and invasion of OSCC cells, at least in part by directly targeting Notch1. These findings suggested that the association between miR-495 and Notch1 may be a potential therapeutic target for the treatment of OSCC.

\section{Acknowledgements}

Not applicable.

\section{Funding}

No funding was received.

\section{Availability of data and materials}

The datasets used and/or analyzed during the present study are available from the corresponding author on reasonable request.

\section{Authors' contributions}

HJ and LL designed this research. LL and QW performed the RT-qPCR, CCK-8 and Matrigel invasion assays. Western blot analysis and the luciferase reporter assay were conducted by YY. All authors have read and approved the final draft.

\section{Ethics approval and consent to participate}

The present study was approved by the Ethics Committee of the Yidu Central Hospital of Weifang (Weifang, China). Written informed consent was obtained from all patients who participated in the present study.

\section{Patient consent for publication}

Not applicable.

\section{Competing interests}

The authors declare that they have no competing interests.

\section{References}

1. Brocklehurst PR, Baker SR and Speight PM: Oral cancer screening: What have we learnt and what is there still to achieve? Future Oncol 6: 299-304, 2010.

2. Hui Y, Li Y, Jing Y, Feng JQ and Ding Y: miRNA-101 acts as a tumor suppressor in oral squamous cell carcinoma by targeting CX chemokine receptor 7. Am J Transl Res 8: 4902-4911, 2016.

3. Pérez-Sayáns M, Suárez-Peñaranda JM, Padin-Iruegas ME, Gayoso-Diz P, Reis-De Almeida M, Barros-Angueira F, Gándara-Vila P, Blanco-Carrión A and García-García A: The loss of p16 expression worsens the prognosis of OSCC. Appl Immunohistochem Mol Morphol 23: 724-732, 2015.

4. Walden MJ and Aygun N: Head and neck cancer. Semin Roentgenol 48: 75-86, 2013

5. Sinha N, Mukhopadhyay S, Das DN, Panda PK and Bhutia SK: Relevance of cancer initiating/stem cells in carcinogenesis and therapy resistance in oral cancer. Oral Oncol 49: 854-862, 2013.

6. Guo T and Califano JA: Molecular biology and immunology of head and neck cancer. Surg Oncol Clin N Am 24: 397-407, 2015.

7. Wu MJ, Jan CI, Tsay YG, Yu YH, Huang CY, Lin SC, Liu CJ, Chen YS, Lo JF and Yu CC: Elimination of head and neck cancer initiating cells through targeting glucose regulated protein78 signaling. Mol Cancer 9: 283, 2010.

8. Hitt R and Echarri MJ: Molecular biology in head and neck cancer. Clin Transl Oncol 8: 776-779, 2006.

9. Kloosterman WP and Plasterk RH: The diverse functions of microRNAs in animal development and disease. Dev Cell 11: 441-450, 2006.

10. Bartel DP: MicroRNAs: Genomics, biogenesis, mechanism, and function. Cell 116: 281-297, 2004.

11. Xie B,Ding Q,Han Hand Wu D: miRCancer: A microRNA-cancer association database constructed by text mining on literature. Bioinformatics 29: 638-644, 2013.

12. An L, Liu Y, Wu A and Guan Y: microRNA-124 inhibits migration and invasion by down-regulating ROCK1 in glioma. PLoS One 8: e69478, 2013.

13. Zhai L, Li Y, Lan X and Ai L: MicroRNA-10a-5p suppresses cancer proliferation and division in human cervical cancer by targeting BDNF. Exp Ther Med 14: 6147-6151, 2017.

14. Huang J, He Y, McLeod HL, Xie Y, Xiao D, Hu H, Chen P, Shen L, Zeng S, Yin X, et al: miR-302b inhibits tumorigenesis by targeting EphA2 via Wnt/ $\beta$-catenin/EMT signaling cascade in gastric cancer. BMC Cancer 17: 886, 2017.

15. Wang YJ, Zhang ZF, Fan SH, Zhuang J, Shan Q, Han XR, Wen X, Li MQ, Hu B, Sun CH, et al: MicroRNA-433 inhibits oral squamous cell carcinoma cells by targeting FAK. Oncotarget 8: 100227-100241, 2017.

16. Wang T, Ren Y, Liu R, Ma J, Shi Y, Zhang L and Bu R: miR-195-5p suppresses the proliferation, migration, and invasion of oral squamous cell carcinoma by targeting TRIM14. Biomed Res Int 2017: 7378148, 2017.

17. Liu B, Chen W, Cao G, Dong Z, Xu J, Luo T and Zhang S: MicroRNA-27b inhibits cell proliferation in oral squamous cell carcinoma by targeting FZD7 and Wnt signaling pathway. Arch Oral Biol 83: 92-96, 2017.

18. Weng J, Zhang H, Wang C, Liang J, Chen G, Li W, Tang H and Hou J: miR-373-3p targets DKK1 to promote EMT-induced metastasis via the Wnt/ $\beta$-catenin pathway in tongue squamous cell carcinoma. Biomed Res Int 2017: 6010926, 2017.

19. Ryan BM, Robles AI and Harris CC: Genetic variation in microRNA networks: The implications for cancer research. Nat Rev Cancer 10: 389-402, 2010.

20. Garzon R, Marcucci G and Croce CM: Targeting microRNAs in cancer: Rationale, strategies and challenges. Nat Rev Drug Discov 9: 775-789, 2010.

21. Wang C, Yun Z, Zhao T, Liu X and Ma X: MiR-495 is a predictive biomarker that downregulates GFI1 expression in medulloblastoma. Cell Physiol Biochem 36: 1430-1439, 2015. 
22. Mao Y, Li L, Liu J, Wang L and Zhou Y: MiR-495 inhibits esophageal squamous cell carcinoma progression by targeting Akt1. Oncotarget 7: 51223-51236, 2016.

23. Chen SM, Chen HC, Chen SJ, Huang CY, Chen PY, Wu TW, Feng LY, Tsai HC, Lui TN, Hsueh C and Wei KC: MicroRNA-495 inhibits proliferation of glioblastoma multiforme cells by downregulating cyclin-dependent kinase 6. World J Surg Oncol 11: 87, 2013.

24. Zhang B, Yuan F, Liu J, Li Y, Zhou F, Liu X, Hao Z, Li Q, Zheng Y and Wang W: Hsa-miR-495 acts as a tumor suppressor gene in glioma via the negative regulation of MYB. Mol Med Rep 14: 977-982, 2016.

25. Jiang $\mathrm{W}$, Zheng $\mathrm{J}, \mathrm{Yu} \mathrm{T}$ and Wang J: Overexpression of microRNA-495 suppresses the proliferation and invasion and induces the apoptosis of osteosarcoma cells by targeting high-mobility group nucleosome-binding domain 5. Oncol Rep 38: 1099-1107, 2017.

26. Livak KJ and Schmittgen TD: Analysis of relative gene expression data using real-time quantitative PCR and the $2^{-\Delta \Delta C_{\mathrm{T}}}$ method. Methods 25: 402-408, 2001.

27. Wang S, Fan H, Xu J and Zhao E: Prognostic implication of NOTCH1 in early stage oral squamous cell cancer with occult metastases. Clin Oral Investig 22: 1131-1138, 2017.

28. Yoshida R, Nagata M, Nakayama H, Niimori-Kita K, Hassan W, Tanaka T, Shinohara M and Ito T: The pathological significance of Notch1 in oral squamous cell carcinoma. Lab Invest 93 : 1068-1081, 2013

29. Gan RH, Wei H, Xie J, Zheng DP, Luo EL, Huang XY, Xie J, Zhao Y, Ding LC, Su BH, et al: Notch1 regulates tongue cancer cells proliferation, apoptosis and invasion. Cell Cycle 17: 216-224, 2018

30. Natsuizaka M, Whelan KA, Kagawa S, Tanaka K, Giroux V, Chandramouleeswaran PM, Long A, Sahu V, Darling DS, Que J, et al: Interplay between Notch1 and Notch3 promotes EMT and tumor initiation in squamous cell carcinoma. Nat Commun 8: 1758, 2017.

31. Zhong R, Bao R, Faber PW, Bindokas VP, Bechill J, Lingen MW and Spiotto MT: Notch1 activation or loss promotes HPV-induced oral tumorigenesis. Cancer Res 75: 3958-3969, 2015.

32. Rastogi B, Kumar A, Raut SK, Panda NK, Rattan V, Joshi N and Khullar M: Downregulation of miR-377 promotes oral squamous cell carcinoma growth and migration by targeting HDAC 9 . Cancer Invest 35: 152-162, 2017

33. Yan Y, Wang X, Ven $\varnothing$ MT, Bakholdt V, Sørensen JA, Krogdahl A, Sun Z, Gao S and Kjems J: Circulating miRNAs as biomarkers for oral squamous cell carcinoma recurrence in operated patients Oncotarget 8: 8206-8214, 2017.
34. Manikandan M, Deva Magendhra Rao AK, Arunkumar G, Manickavasagam M, Rajkumar KS, Rajaraman R and Munirajan AK: Oral squamous cell carcinoma: microRNA expression profiling and integrative analyses for elucidation of tumourigenesis mechanism. Mol Cancer 15: 28, 2016.

35. Yan L, Yao J and Qiu J: miRNA-495 suppresses proliferation and migration of colorectal cancer cells by targeting FAM83D. Biomed Pharmacother 96: 974-981, 2017.

36. Bai Z, Wang J, Wang T, Li Y, Zhao X, Wu G, Yang Y, Deng W and Zhang Z: The miR-495/Annexin A3/p53 axis inhibits the invasion and EMT of colorectal cancer cells. Cell Physiol Biochem 44: 1882-1895, 2017.

37. Eun JW, Kim HS, Shen Q, Yang HD, Kim SY, Yoon JH, Park WS, Lee JY and Nam SW: MicroRNA-495-3p functions as a tumor suppressor by regulating multiple epigenetic modifiers in gastric carcinogenesis. J Pathol 244: 107-119, 2018

38. Li Z, Cao Y, Jie Z, Liu Y, Li Y, Li J, Zhu G, Liu Z, Tu Y, Peng G, et al: miR-495 and miR-551a inhibit the migration and invasion of human gastric cancer cells by directly interacting with PRL-3. Cancer Lett 323: 41-47, 2012.

39. Wang $\mathrm{H}$, Jiang Z, Chen $\mathrm{H}$, Wu X, Xiang $\mathrm{J}$ and Peng J: MicroRNA-495 inhibits gastric cancer cell migration and invasion possibly via targeting high mobility group AT-Hook 2 (HMGA2). Med Sci Monit 23: 640-648, 2017.

40. Li JZ, Wang ZL, Xu WH, Li Q, Gao L and Wang ZM: MicroRNA-495 regulates migration and invasion in prostate cancer cells via targeting Akt and mTOR signaling. Cancer Invest 34: 181-188, 2016.

41. Xu YY, Tian J, Hao Q and Yin LR: MicroRNA-495 downregulates $\mathrm{FOXC1}$ expression to suppress cell growth and migration in endometrial cancer. Tumour Biol 37: 239-251, 2016.

42. Lv C, Bai Z, Liu Z, Luo P and Zhang J: MicroRNA-495 suppresses human renal cell carcinoma malignancy by targeting SATB1. Am J Transl Res 7: 1992-1999, 2015.

43. Tan M, Mu X, Liu Z, Tao L, Wang J, Ge J and Qiu J: microRNA-495 promotes bladder cancer cell growth and invasion by targeting phosphatase and tensin homolog. Biochem Biophys Res Commun 483: 867-873, 2017.

44. Cao M, Nie W, Li J, Zhang Y, Yan X, Guan X, Chen X, Zen K, Zhang CY, Jiang X and Hou D: MicroRNA-495 induces breast cancer cell migration by targeting JAM-A. Protein Cell 5: 862-872, 2014

45. Zou Z, Zou R, Zong D, Shi Y, Chen J, Huang J, Zhu J, Chen L, Bao X, Liu Y, et al: miR-495 sensitizes MDR cancer cells to the combination of doxorubicin and taxol by inhibiting MDR1 expression. J Cell Mol Med 21: 1929-1943, 2017.

46. Hwang-Verslues WW, Chang PH, Wei PC, Yang CY, Huang CK, Kuo WH, Shew JY, Chang KJ, Lee EY and Lee WH: miR-495 is upregulated by E12/E47 in breast cancer stem cells, and promotes oncogenesis and hypoxia resistance via downregulation of E-cadherin and REDD1. Oncogene 30: 2463-2474, 2011. 